\title{
Typographie et rhétorique du lisible. Les créations de David Carson
}

\author{
Christian Vandendorpe
}

Université d'Ottawa

\begin{abstract}
Alors que le $\mathrm{XIX}^{\mathrm{e}}$ siècle avait soumis le texte au choc de la mécanisation massive, la seconde moitié du $\mathrm{XX}^{\mathrm{e}}$ siècle le soumet à celui de la virtualisation, l'émancipant de son support d'encre et de papier en faveur de pixels qui papillotent sur un écran. L'écriture manuscrite semble vouée à disparaître tandis que le texte entre dans l'ère d'une sur-norme typographique.

C'est dans ce contexte qu'apparaissent les travaux de David Carson. A priori, rien ne le prédisposait à se lancer en typographie. Né en 1956, il avait d'abord fait des études de sociologie et enseigné au secondaire tout en se livrant à du surf de compétition sur les plages de Californie. Il découvre la typographie et la mise en page lors d'un cours d'été en 1980 et, tout en gardant son poste d'enseignant jusqu'en 1987, il s'engouffre dans ce nouveau domaine avec une passion peu commune. Il consacre ses étés à des conférences sur le graphisme, d'abord aux Etats-Unis, puis en Suisse, où il rencontre Hans-Rudolf Lutz, qui exercera sur lui une influence qu'il reconnaît volontiers. En 1989, il obtient la direction artistique d'un nouveau magazine consacré au surf, Beach Culture. Entouré d'une équipe de graphistes, il expérimente de façon sauvage, enfreignant toutes les règles de l'art typographique et de la mise en page. Malheureusement, la présentation chaotique du magazine fera fuir la publicité, de telle sorte que le magazine devra cesser de publier en 1991.
\end{abstract}


En 1992, Carson obtient la direction artistique de Ray Gun, un magazine de musique rock qui tire à 150000 exemplaires et où il restera jusqu'en 1995, date où il retourne à la publicité. Nombre de ses créations sont présentées et discutées dans The end of print ${ }^{1}$ et $\mathrm{Da}$ vid Carson: 2ndsight ${ }^{2}$, deux ouvrages réalisés avec le graphiste anglais Lewis Blackwell, et dont Carson a conçu la maquette. Le simple fait de feuilleter ces ouvrages donne déjà une bonne idée de sa façon de concevoir la typographie.

Celle-ci est aux antipodes de la conception classique que Maximilien Vox avait résumée dans son aphorisme : «En typographie, il n'y a qu'un seul degré de bien : la perfection $»^{3}$.
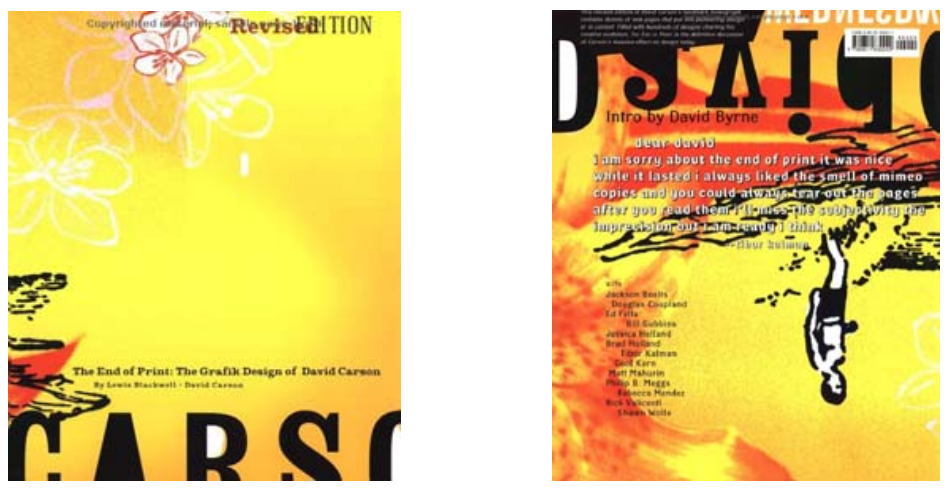

Figures 1 et 2. Couvertures recto et verso de The end of print. Édition révisée (2000)

\footnotetext{
${ }^{1}$ The end of print. San Francisco, Chronicle Books, 1995. Édition révisée en 2000. Cet article fait référence à la première édition, à l'exception de la page couverture. Cet ouvrage en est maintenant à sa cinquième édition et s'est vendu à plus de 125000 exemplaires.

2 David Carson : 2ndsight. Grafik Design After the End of Print. New York, Universe Publishing, 1996 ( $2^{\mathrm{e}}$ édition en 1997). Un autre ouvrage à consulter sur cette question est le collectif dirigé par Kuipers D., Ray Gun : Out of Control, Simon \& Schuster, 1997.

${ }^{3}$ Cité en épigraphe à De plomb, d'encre et de lumière, sous la dir. de Gid R., Paris, Imprimerie nationale, 1982.
} 
Dès la page couverture de The end of print, la disposition des mots et des images est déjà un indice de cette rupture avec les normes en vigueur (figures 1 et 2). En tournant les pages, on découvre que rien n'a été épargné de l'idéal typographique de perfection et de lisibilité : le faux-titre est presque entièrement masqué par un collage; la date de publication est introuvable; le livre ne comporte ni titre courant ni numérotation des pages. Surtout, la maquette d'ensemble ne présente aucune régularité : certaines pages offrent une marge supérieure très large alors que la plupart ne laissent aucun espace entre le bord et le texte. Il en va de même pour la disposition des colonnes, dont l'allée est parfois réduite à zéro. L'interligne est tout aussi erratique et il est fréquent de voir des lignes se chevaucher. La ligne de texte est parfois rompue en échelles et les espacements entre les lettres sont souvent irréguliers. Pour faire bonne mesure, Carson va jusqu'à oblitérer le texte par des taches ou des collages, le découper ou l'imprimer partiellement sur fond noir. Les tortures qu'il impose au texte ne s'arrêtent pas là : il lui arrive d'inverser la séquentialité de blocs de textes, d'intervertir une ligne sur deux ou de désorganiser des fragments de mots qu'il a préalablement découpés (figure 3 ).

\section{MIS - DON'T \\ LEGI- TAKE \\ FOR BILITY \\ ICATIO N. CO MMUN}

Figure 3. «Don't mistake legibility for communication » (« Ne confondez pas lisibilité et communication »). Extrait de The end of print.

Une phrase peut aussi être brutalement interrompue et continuée après quelques sauts de ligne. Ou encore, Carson dispose sur trois colonnes justifiées et espacées par une allée des lignes d'un texte qu'il faut lire en continu, soit la première ligne de la colonne de 
gauche suivie de la première ligne de la colonne voisine et ainsi de suite (figure 4).

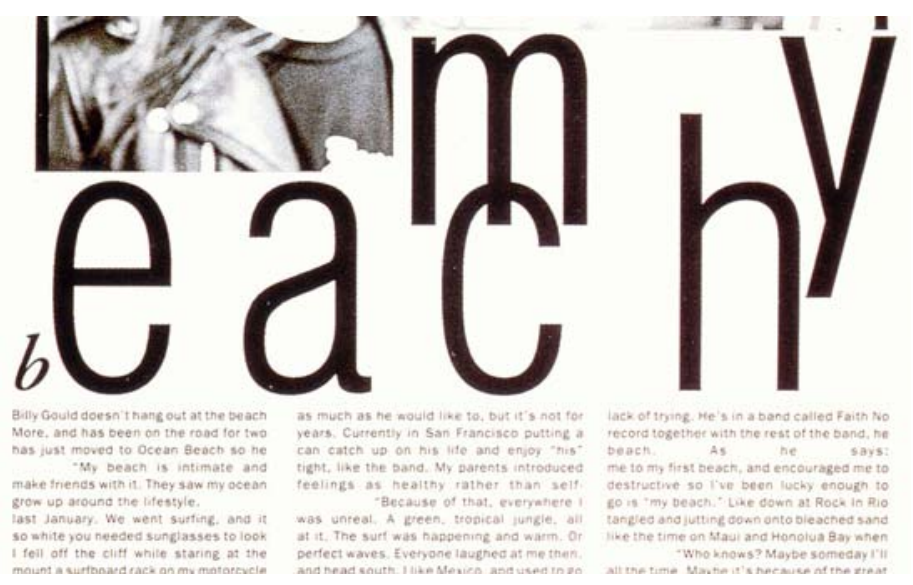

Figure 4. Fausses colonnes de texte (The End of print)

Il arrive aussi que des blocs de texte soient disposés de guingois ou que les mots soient éparpillés sur la page. Un autre procédé est de superposer deux textes en les décalant légèrement, afin de produire un effet de bougé. La police de caractère est d'une grande irrégularité, des tailles de 36 points alternant dans un même texte avec du 8 points. Parfois, un bloc de texte est extrudé de la colonne de texte selon une forme définie, pour laisser place, par exemple, à la silhouette d'un personnage ou à un point d'interrogation, et ce morceau de texte est disposé sur la colonne ou la page voisine (figure $5)$. 

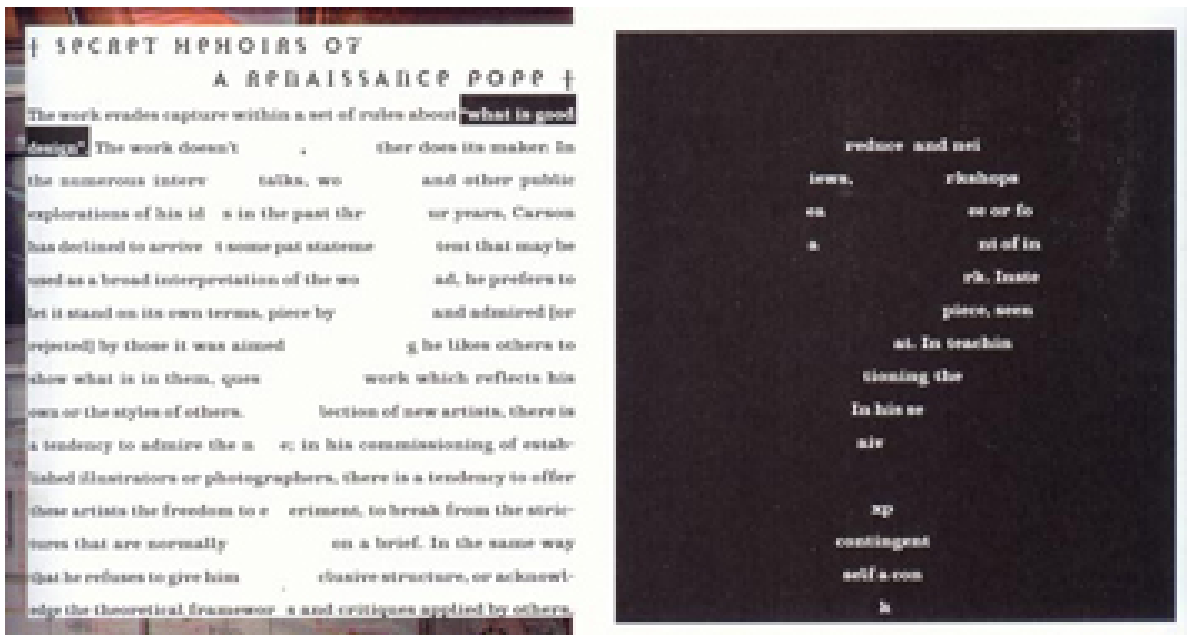

Figure 5. Un cas de dimorphisme textuel (Extrait de The end of print)

Certaines lignes sont systématiquement barrées, comme pour dissuader le lecteur de les lire. Parfois la colonne de texte est justifiée à droite, mais en drapeau à gauche. Les césures sont le plus souvent aberrantes. La composition de longs passages en lettres capitales sans interligne vient encore compliquer la tâche de lecture. Tous ces procédés sont familiers chez Carson depuis la fin des années 80 . Mais la désorientation du lecteur atteint sans doute un sommet dans un numéro de Beach Culture où le sommaire est disposé sous la forme d'un texte composé à la verticale et replacé à l'horizontale. Pour se le représenter, on peut penser au calligramme "Pluie" d'Apollinaire auquel on aurait imposé une rotation de quelque 90 degrés vers la droite (figure 6). 


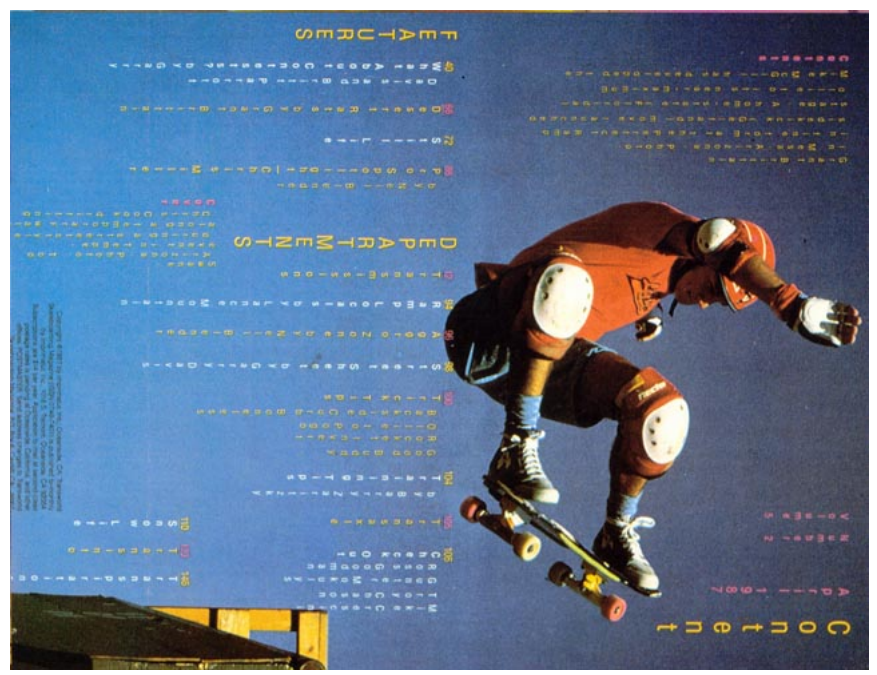

Figure 6. Un sommaire de Beach Culture.

Bref, on peut difficilement imaginer textes soumis à plus d'avanies, torturé jusqu'à la limite de la lisibilité.

Les illustrations ne sont pas traitées avec plus de respect, Carson multipliant les images déformées, les «coups de poing » visuels, avec une prédilection pour le flou et le polaroïd. Loin de rechercher la précision du cadrage, il multiplie les perspectives tronquées, les images confuses, retravaillées avec force airbrush sous Photoshop. Il arrive aussi qu'un cadre vide de grande taille soit placé au milieu d'un article, avec en grosses lettres le message « (((sorry))) no graphix » afin de souligner ironiquement l'absence d'illustration. Bref, au lieu de l'esthétique léchée du magazine et de sa maquette tirée au cordeau, Carson a choisi l'esthétique du graffiti : il utilise la typographie, les images et la couleur à la façon des artistes de rue qui vaporisent de la peinture en aérosol pour couvrir de tags et de graffiti l'espace urbain. 


\section{Automatismes et lisibilité}

La première impression que suscitent ces pages est que l'on se trouve devant une série de tableaux colorés ou de marques erratiques plutôt que devant du texte. La lecture, en effet, ne s'enclenche pas spontanément, tant nos automatismes de lecture sont pris à revers par un texte qui diffère radicalement de sa mise en forme habituelle. Le plus souvent, il est même difficile de déterminer où commence le texte, comment s'enchaînent les lignes, et si les brusques changements de police ne sont pas des signes aléatoires abandonnés par un enfant qui aurait joué avec des lettres, plutôt que la manifestation d'une intention de communiquer.

Toutefois, le lecteur qui scrute un peu la page pourra être arrêté par des mots çà et là, et ce sera parfois suffisant pour lui donner envie de regarder de plus près et enclencher un travail de lecture. Celui-ci ne manque pas d'être ardu, comme le déchiffrement d'un rébus, car il faut arracher chaque mot à la gangue opaque d'une typographie irrégulière et que masquent souvent des images. La lecture devient ainsi une lente et difficile conquête du sens sur les divers procédés dont le typographe a usé pour tromper nos habitudes et nous empêcher de lire aisément. Pour mieux empêcher toute mise en place d'une routine, chaque page réserve son lot de surprises graphiques. Il y a cependant un système derrière les choix de mise en page. Carson affirme en effet avoir lu chaque article afin d'en donner une interprétation adéquate: " Je lis le texte et ensuite j'essaie de l'interpréter $»{ }^{4}$; les articles qui ne l'inspirent pas sont composés de façon classique, ce qui peut être perçu par leurs auteurs comme un désaveu $^{5}$. De fait, un examen attentif des maquettes permet de dégager des liens entre les choix de typographie et le sujet des articles ou de certaines phrases. Au chaos apparent succède alors une quête ludique des rapports signifiant-signifié.

À titre d'exemple, dans Beach culture, un article sur l'enseignement du surf à des aveugles s'ouvre sur une double page intégralement

\footnotetext{
4 «I read the copy and then I try to interpret it », The end of print, non paginé.

${ }^{5}$ Clark J., «Illegible David Carson cannot not communicate », http://www.joeclark.org/davidcarson.html
} 
noire : la confusion du lecteur et la perte de repères visibles constitue sans aucun doute une pertinente introduction au sujet traité. Inversement, un des exercices que Carson propose dans ses ateliers est de concevoir une maquette qui soit totalement inappropriée pour un article donné, comme de présenter un article sur les sans-abris à la façon d'un article de revue de mode.

On a vu aussi, dans la figure 3 («Don't mistake legibility for communication ») comment Carson pouvait parfois découper les mots et en altérer la continuité graphématique pour forcer le lecteur à un véritable travail de décodage. Ici encore, le recouvrement du sens par la typographie est impossible à ignorer. Le feuilletage de ces albums encourage une exploration du texte comme une série de calligrammes, faisant renouer le lecteur avec le vieux rêve d'une langue hiéroglyphique ou mimétique, qui poursuit l'humanité depuis le Cratyle de Platon ${ }^{6}$. Mais, à la différence de bien des tentatives antérieures, le graphiste ne prétend pas inventer un nouvel alphabet : il se contente d'en modifier la disposition habituelle.

Carson est évidemment conscient des problèmes de lisibilité que pose cette esthétique. Si la mise en page de Beach Culture lui a valu plus de 150 prix $^{7}$, elle a aussi révulsé les publicitaires, à tel point que le magazine a dû fermer ses portes après seulement six numéros. De même, le magazine Ray Gun finira par se séparer de lui après quelques années.

Pourtant, Carson ne recule pas sur la notion du lisible et il attaque à son tour l'intérêt de cette notion par rapport à une économie de la communication. Plus précisément, il reproche aux tenants de la lisibilité de négliger les émotions. Or, celles-ci ont pour lui un rôle essentiel, et il lui importe peu d'être lisible si aucune émotion n'est communiquée. C'est la recherche de cet effet, de cette réaction émotionnelle, qui l'intéresse au premier chef: "Vous pouvez être lisible, mais quelle émotion y a-t-il dans le message? C'est cela qui est important pour moi [...] Quand je regarde une page dans un

\footnotetext{
${ }^{6}$ Genette G., Mimologiques, Paris, Éditions du Seuil, Coll. Points, 1976.

${ }^{7} \mathrm{http}$ ://www.fontshop.de/typo98/enhanced/sprecher/e_carson.html
} 
livre, un magazine, une œuvre graphique, je veux une réaction affective $»^{8}$.

En attirant ainsi l'attention sur ses procédés, la typographie cesse de se confondre avec un idéal de pure transparence au service du texte et devient spectacle. Elle s'affirme comme un langage capable de jouer sur divers plans et de susciter des effets. Alors qu'elle excellait dans le registre de la précision et de la perfection formelle depuis qu'à Venise Alde Manuce avait créé ses magnifiques polices de caractères, l'art du graphiste s'annexe désormais ces autres registres que sont l'obscurité calculée et le calembour visuel.

Le trajet accompli par la typographie en un demi-millénaire n'est donc pas sans évoquer celui qu'a parcouru la rhétorique depuis l'époque classique. Là où l'Antiquité cultivait les figures propres à assurer la production du sublime ${ }^{9}$, la révolution romantique avait donné des lettres de noblesse à tous les procédés, quels qu'ils soient. Après un Victor Hugo qui se targuait d'avoir « mis un bonnet rouge au Dictionnaire » («Plus de mot sénateur! plus de mot roturier ! $\rangle^{10}$ ), un Raymond Queneau explorera avec bonheur les jeux sur toutes les faces du signifiant, ne reculant, dans ses Exercices de style et autres Zazie, devant aucune apocope, syncope ou crase, et prenant plaisir à recontextualiser en langue écrite des formes issues de tournures orales ou populaires. Aujourd'hui, la riche production du genre comique continue d'explorer toutes les possibilités de déplacement et de substitution qu'offre le signifiant linguistique.

Une semblable évolution est à l'œuvre dans la mise en forme du texte. Surfant sur la vague de remise en question des valeurs que le courant de la déconstruction et du postmodernisme a engendré aux Etats-Unis, Carson revendique pour la typographie le droit de jouer avec les signes eux-mêmes, sous toutes leurs formes, y compris à

\footnotetext{
${ }^{8}$ "You may be legible, but what is the emotion contained in the message? That is important to me.[...] When I turn to a page in a book, to a magazine, to any graphic design, I want an emotional reaction.» Extrait de The End of Print.

${ }^{9}$ Longin, Du Sublime, $\mathrm{I}^{\mathrm{er}}$ siècle.

${ }^{10}$ Hugo V., « Réponse à un acte d'accusation, Les Contemplations.
} 
l'état de brouillons, de biffures ou de raclures de graphisme. Et cela, dans une mise en page chaotique et systématiquement incohérente - ou, plus exactement, dépourvue de repères stables. Ce faisant, le graphiste attire assurément l'attention du lecteur sur la typographie et tend même à hausser celle-ci, du rang de simple medium technique, à celui de langage artistique. On sait en effet que le brouillage systématique de la forme est un procédé de la création de l'effet artistique. Il avait été mis à nu dès le début du $\mathrm{XX}^{\mathrm{e}}$ siècle par l'école formaliste russe. Selon Chklovski :

...le caractère esthétique se révèle toujours par les mêmes signes : il est créé consciemment pour libérer la perception de l'automatisme; sa vision représente le but du créateur et elle est construite artificiellement, de manière à ce que la perception s'arrête sur elle et arrive au maximum de sa force et de sa durée. ${ }^{11}$

La rhétorique du lisible que Carson met en place joue principalement sur les effets de parasitage du texte par la typographie et celleci est le premier outil qu'il va utiliser afin de produire sur le public le choc publicitaire associé à une marque. Car, pour lui, les mots sont d'abord des entités visuelles : " Les mots sont des images, eux aussi $[\ldots .$.$] . Et ils véhiculent un impact affectif bien avant d'avoir$ été véritablement lus $»^{12}$.

Il n'est pas surprenant que ces expériences soient apparues dans des revues qui s'adressent à des jeunes dont l'univers auditif est saturé de musique pop et de rock and roll. Nous avons tous entendu de ces chansons qui mixent paroles et musique de façon à donner à cette dernière une telle prépondérance que le propos en devient souvent inaudible. Et cela importe peu, car le message n'est pas dans le discours, mais dans son brouillage. De même, Carson écrase parfois tellement le texte sous le vacarme des procédés typographiques que celui-ci en devient presque illisible. Il y a toutefois une différence

\footnotetext{
${ }_{11}^{11}$ Cité par Todorov T., Théorie de la littérature, Paris, Seuil, 1965, p. 94.

12 «Words are images, too, and sit within pages or onscreen as parts of larger images. And they carry an emotional impact long before they're actually read », Extrait de Carson : 2ndSight (non paginé).
} 
notable avec le monde de la chanson, car celle-ci emprisonne l'auditeur dans la fugacité de l'instant, alors que le l'œil permet toujours au lecteur de saisir diverses composantes de l'image dans de brusques épiphanies.

Cette rhétorique typographique vise donc moins à empêcher de lire qu'à désautomatiser ${ }^{13}$ les procédés de lecture : elle transpose au plan de la mise en page des procédés que le langage poétique a faits siens depuis toujours et qui visent à détourner l'attention sur la forme de l'expression en faisant échec à une compréhension directe des idées dans l'abolition du langage. Un média est en effet toujours susceptible de devenir «invisible» tant l'esprit s'attache d'abord au sens. Il en va de même pour ce média par excellence qu'est le langage, au point que des individus bilingues peuvent souvent ne plus savoir dans quelle langue ils ont lu un ouvrage ou écouté une conférence. La rhétorique visera à éviter cet écueil en répertoriant les écarts - au plan du lexique, de la syntaxe ou des types d'images utilisées - susceptibles de laisser une empreinte durable sur l'esprit des auditeurs et de transformer la prose en spectacle.

Une telle opération sur la typographie a été longtemps impensable car celle-ci est conçue comme un média neutre, une technique, au service du texte et de la lisibilité. Tout effet de brouillage sur ce premier niveau de perception est susceptible de faire scandale dans une civilisation où la multiplication des signes écrits a fait de la lecture, pour la plus grande partie de la population, une activité quasiment permanente et inévitable, à tel point qu'elle confine souvent à une servitude sémiotique plutôt qu'à une activité discrète et délibérée.

Il est à noter que cette rhétorique du choc visuel et du brouillage perceptif est très proche de l'esthétique publicitaire. Plusieurs grandes entreprises ne s'y sont d'ailleurs pas trompées, qui se disputent

\footnotetext{
${ }^{13}$ Sur la question des automatismes en lecture, voir Vandendorpe C., « La lecture entre automatisation et déchiffrement », dans L'Acte de lecture, sous la dir. de Saint-Jacques D., Québec, Nuit Blanche, 1994, p. 213-228.
} 
depuis dix ans les services de Carson : Giorgio Armani, Microsoft, Lucent Technologies, Nike, Pepsi, Xerox et autres. Et les agences de création publicitaire l'invitent à donner des conférences et des ateliers dans le monde entier.

Dans une affiche qui lui avait été commandée en 1992 pour la voiture Camaro, mais qui n'a pas été utilisée, le graphiste prendra le risque de jouer sur les seuls procédés typographiques: casse, chasse, interligne, taille des caractères. Il en résulte un effet de désorientation visuelle qui, même s'il ne dure pas, est apte à laisser une trace dans la mémoire du lecteur, trace idéalement accompagnée du sentiment de satisfaction que donne au lecteur la résolution de ce qui, à première vue, pouvait ressembler à une figure illisible ou à un rébus (figure 7).

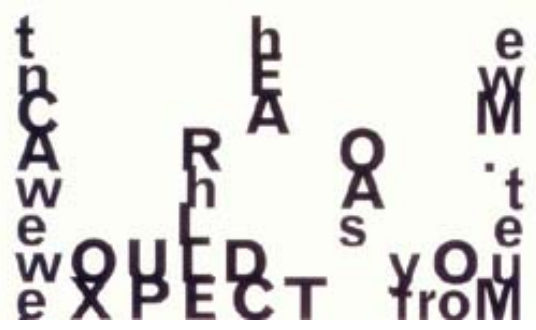

Figure 7. Partie supérieure d'un projet d'annonce pour la Camaro. (The end of print)

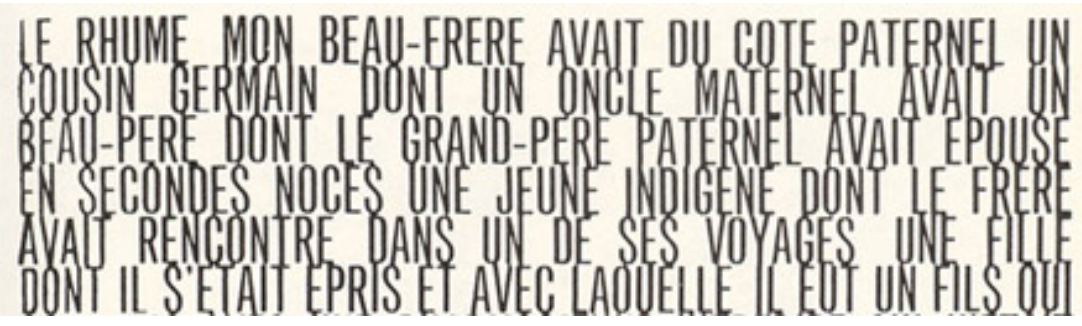

Figure 8. Massin : Le récit du pompier dans Ionesco, la Cantatrice chauve 
En soi, le procédé n'est pas neuf et Massin l'avait déjà utilisé pour la mise en page d'un extrait de Ionesco (figure 8).

Cette influence de l'esthétique publicitaire sur la maquette des magazines est également à l'œuvre, mais d'une autre manière, chez Neville Brody, un graphiste anglais souvent comparé à David Carson. Comme ce dernier, Brody a été directeur artistique d'un magazine de musique populaire, The Face, et il croit, lui aussi, que la typographie doit produire un effet émotif. Il explique ainsi s'être attaché à produire des compositions dans lesquelles « toute marque apposée sur la page était une réponse émotive ou une extension logique des idées exprimées $\gg{ }^{14}$. Au plan typographique, il a surtout joué avec les titres, qu'il aime intégrer dans une structure caractéristique du logo (figure 9).

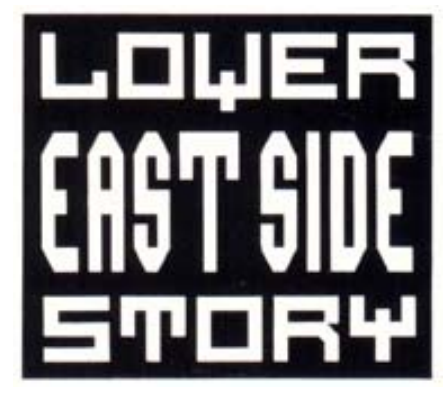

Figure 9. Neville Brody. Titre d'un article de Tom Walts, The Face, $n^{\circ} 67$, novembre 1985. Le caractère a été dessiné par Brody spécialement pour cet usage. Extrait de Jon Wozencroft, The Graphic Language of Neville Brody, Thames and Hudson, 1988, p. 31.

Le mouvement par lequel la typographie devient spectacle n'est donc pas un phénomène de génération spontanée. Dans un article consacré à David Carson, Rick Poynor a mis en évidence tout ce que celui-ci doit à l'esthétique de la télévision style MTV, ainsi qu'à des pionniers du graphisme postmoderne, tels April Greiman

14 «Every single mark on the page was either an emotive response or a logical extension of the ideas $»:$ http://www.artandculture.com/arts/artist?artistId=1208 
et Rudy VanderLans du magazine Emigré ${ }^{15}$. Il ne faudrait pas oublier non plus la Cranbrook Academy of Art, prestigieuse école de graphisme où, dès le début des années 80 , Katherine McCoy et ses étudiants avaient mis en place une théorie de la «typographie comme discours ${ }^{16}$. Ce qui est nouveau, c'est la pénétration dans des médias de masse de procédés jusque-là confinés aux œuvres de création et réservés à des milieux d'avant-garde.

Le magazine Wired, dont le premier numéro contenait d'ailleurs un article sur Ray Gun et David Carson ${ }^{17}$, illustre le succès de ce nouveau courant dans les années 90 . Même si le graphisme agressif en est parfois gratuit et peut gêner la lecture, il a contribué à donner à ce magazine un style distinctif et a permis de remarquables rencontres entre le support imprimé et le sujet traité, comme cet article de Jeff Howe qui, pour démontrer les dérives de la licence numérique, oblige le lecteur à découper, pour pouvoir le lire, le «Reader license agreement » qui sert de préambule à l'article ${ }^{18}$.

L'esthétique de la typographie spectacle tend maintenant à déborder l'univers de publicité et du magazine pour s'étendre au monde du livre. C'est le cas, par exemple, de l'album Ray Gun : Out of Control, où le lecteur doit y regarder à deux (ou trois) fois avant de découvrir la pagination, tant celle-ci est subtilement intégrée au contenu, comme des incrustations à demi effacées sur un espace coloré et graphiquement saturé. La maison londonienne de livres d'art Phaidon a aussi parfois recours à divers procédés de déni typographique pour la couverture de ses livres (figures 10 et 11).

15 Poynor R., «Alternative by Design? », Ray Gun. Out of control, Simon \& Schuster, 1997, p. 226-234.

${ }^{16} \mathrm{http}: / / \mathrm{ndm}$. si.edu/EXHIBITIONS/mixingmessages/essay/typo/t_b.13.5.html

${ }^{17}$ Patton P., « A new breed of Designer», Wired 1.1, mars 1993.

${ }^{18}$ Wired, 9.10 , octobre 2001, p. 140-147. 

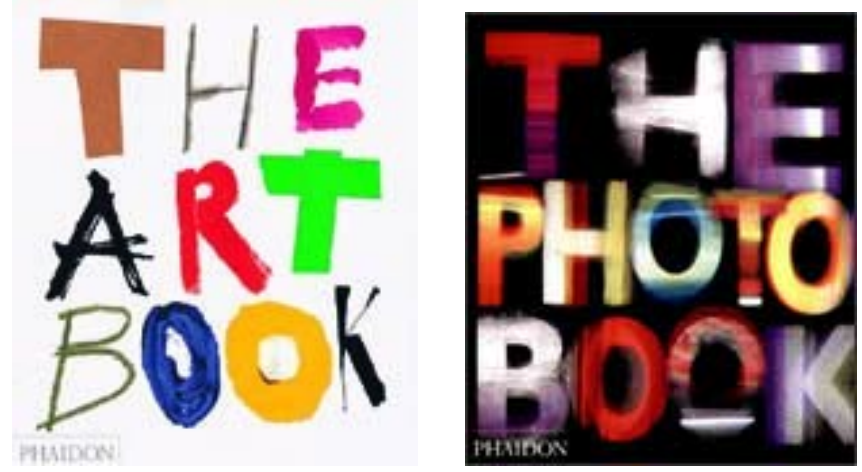

Figures 10 et 11. Page couverture de The Art Book et The Photo Book, Phaidon, 1994 et 1997.

Lorsqu'en 1997, le photographe Albert Watson a demandé à David Carson de faire la maquette de Cyclops $^{19}$, un album sur son parcours de photographe, on est effectivement entré dans une nouvelle ère, où le graphiste est en mesure de marquer de son style la maquette d'un livre, tout comme un couturier met sa griffe sur une collection de vêtements. La signature n'est plus réservée à l'auteur du texte ou à son illustrateur, mais peut désormais s'étendre à l'expert typographe.

\section{Mallarmé et une poétique de l’obscurité}

Il peut paraître excessif d'évoquer Mallarmé à propos de Carson, tant ces deux personnages diffèrent dans leur esthétique. Pourtant, ils ont en commun d'utiliser la typographie en vue de brouiller l'établissement d'une lecture routinière et cantonnée au monde des mots. Mais Carson commence son travail de création là où Mallarmé avait achevé le sien.

\footnotetext{
${ }^{19}$ Bulfinch Press, 1997. Le titre n'apparaît pas sur la page couverture, mais seulement à l'endos.
} 
En 1896, Marcel Proust, âgé de vingt-cinq ans et encore inconnu, publiait dans La Revue blanche un article intitulé "Contre l'obscurité ». Dans ce texte programmatique, il s'attaquait à l'esthétique fin de siècle alors dominante et qui se manifestait par une double obscurité dans les œuvres : celle des idées et des images d'une part, celle de la grammaire de l'autre. Quelques semaines plus tard, Stéphane Mallarmé, de trente ans son aîné et chef de file du mouvement symboliste ainsi attaqué, lui répondra par un article dans la même revue intitulé «Le mystère dans les lettres ». Dans ce texte il revendique pour l'écrivain le droit d'être obscur et il impute toute incompréhension éventuelle à un défaut de lecture : " Je préfère, devant l'agression, rétorquer que des contemporains ne savent pas lire ». Du même souffle, il affirme l'importance fondamentale du blanc sur la page et réclame pour le signe écrit une part dans la production de l'effet poétique : "Je sais, on veut à la Musique, limiter le Mystère; quand l'écrit y prétend. ${ }^{20}$ Un an plus tard, il publiera la première version de «Un coup de dés », poème dont on s'accorde généralement à reconnaître qu'il a fait entrer la poésie dans un nouvel espace : celui de la matérialité du signe typographique ainsi que de la page et du blanc ${ }^{21}$.

En un sens, ce débat entre Mallarmé et Proust ne manque pas d'ironie, car si pour le premier « tout, au monde, existe pour aboutir à un livre $»^{22}$, le second a fait de son livre un véritable univers dans lequel il a transmuté durant quinze ans les divers événements de sa vie : passions, souvenirs, personnages, œuvres et tableaux aimés, façons de parler, lieux visités. Mais Proust croyait passionnément à la capacité analytique du langage et à la possibilité de tout exprimer, fût-ce par des métaphores ou des phrases sinueuses et ramifiées à l'extrême qui s'alignent au fil de paragraphes massifs dans

\footnotetext{
${ }^{20}$ Mallarmé S., Euvres, textes établis par Favre Y.-A., Paris, Garnier, 1985, « Le mystère dans les lettres ». p. 304.

${ }^{21}$ Pour une analyse de ce poème, voir notamment Christin A.-M., « De l'espace typographique à l'écriture du blanc », dans L'aventure des écritures : la page, sous la direction de Zali A., Paris, Bibliothèque nationale de France, 1999; Garron I., «La part typographique », Communication et langages, $\mathrm{n}^{\circ}$ 134, décembre 2002, p. 58-74.

${ }^{22}$ Ibidem. « Quant au Livre », p. 294.
} 
un monument élevé à la linéarité du narratif et que doit mettre en valeur une typographie parfaitement neutre et transparente ${ }^{23}$. Mallarmé, au contraire, opte pour une prose fragmentée, avec des paragraphes qui ne comptent souvent qu'un ou deux mots. Loin de chercher la clarté dans l'expression, il manifeste une conscience aiguë de la part d'ombre que recèle chaque être et n'a pas pour ambition de la dire, mais seulement d'y chercher l'encre dont il éclaboussera le blanc de la page et qui engagera son lecteur dans une lente et difficile démarche de conquête du sens. En cela, son projet disait déjà la nécessité d'un au-delà - ou d'un en-deçà - du langage, qu'il trouvera dans la typographie après l'avoir cherché dans les sonorités du vers et le jeu de la syntaxe.

Cette méfiance du poète à l'égard du langage ordinaire est le produit d'un siècle où la mécanisation du texte avait fait des progrès extraordinaires. En quelques décennies, le passage de la presse à cylindre à la rotative avait permis de porter la production de 500 copies à l'heure en 1811 à 12000 en 1850 . L'imprimé désormais était partout: ce qui avait été jadis une denrée rare et précieuse devenait omniprésent, mais aussi d'une navrante banalité. Et cela devait exalter par contrecoup une recherche forcenée du style, accompagnée de la hantise du cliché, laquelle était devenue une véritable obsession, notamment chez Flaubert. Tout artiste cherche à se démarquer à sa façon du discours social : celle de Mallarmé est de torturer la syntaxe ou de faire intervenir la matérialité du signe et de la page pour démultiplier les effets de sens. Il casse ainsi les habitudes engendrées par une lecture rapide et mécanique : «au sujet de brochures à lire d'après l'usage courant, je brandis un couteau, comme le cuisinier égorgeur de volailles ${ }^{24}$.

Mallarmé décédera l'année suivante, mais son coup de force aura des répercussions importantes. Point tournant dans le rapport au langage poétique, son œuvre servira de repère aux divers mouve-

\footnotetext{
${ }^{23}$ Proust insistera souvent auprès de son éditeur pour que son roman soit imprimé dans un caractère d'un corps très lisible (voir Tadié J.-Y., Marcel Proust : biographie, Paris, Gallimard, 1996). En revanche, l'éditeur introduira de nombreux alinéas qui seront éliminés dans la seconde édition de la Pléiade.

${ }^{24}$ Ibidem. « Quant au Livre », p. 297.
} 
ments d'avant-garde qui expérimenteront avec le calligramme et la typographie, tels Apollinaire, le mouvement futuriste de Marinetti et le dadaïsme, entre 1916 et 1923 . Ces productions resteront toutefois confinées à une période assez brève. Comme le note Johanna Drucker, qui a étudié de près les créations typographiques de cette période, cet extraordinaire bouillonnement sera bientôt marginalisé par la montée du mouvement surréaliste. André Breton rendra la primauté au texte en délaissant les jeux typographiques, parce qu'ils distraient le lecteur, et optera pour une typographie aussi transparente que possible ${ }^{25}$. Comme le note encore Johanna Drucker, tout semble indiquer que «le texte littéraire ne tolère aucune interférence ou manipulation visuelle susceptible de troubler l'énonciation linguistique du matériau verbal $»^{26}$.

Les expérimentations avec la matérialité du support typographique reprendront cependant avec une vigueur nouvelle après 1945. On voit alors apparaître le mouvement lettriste avec Isidore Isou en France et la poésie concrète en Allemagne avec Eugen Gomringer. Celle-ci s'épanouit aussi au Brésil en 1958 avec Decio Pignatari et Augusto de Campos, lui-même lié au monde de la publicité. En France, le mouvement est représenté par Pierre Garnier. En 1968, celui-ci dresse un état de la situation qui ne laisse pas d'être impressionnant. Il attribue l'intérêt du public pour ce type de création à une «mutation qui depuis un siècle s'est emparée du monde : passage de l'intellectualisme au sensoriel, du lisible au visible, du raisonné au sensible $»^{27}$.

\section{L'ordinateur et le chant du signe typographique}

Le projet de Carson arrive à un moment où la mécanisation du signe écrit a franchi une étape supplémentaire par rapport à la situation qui régnait un siècle auparavant. Les vitrines des kiosques sont envahies par des milliers de magazines interchangeables, à la typo-

\footnotetext{
${ }^{25}$ Drucker J., The Visible Word. Experimental Typography and Modern Art, 19091923, Chicago, The University of Chicago Press, 2000, p. 224.

${ }^{26}$ Op. cit., p. 95.

${ }^{27}$ Garnier P., Spatialisme et poésie concrète, paris, Gallimard, 1968, p. 57.
} 
graphie impeccable, souvent dépourvus d'identité visuelle. Surtout, l'ordinateur est devenu incontournable dans la production du texte, reléguant l'écriture manuscrite à des usages marginaux. Même la correspondance privée passe maintenant par Internet, faisant disparaître des messages - dans le premier âge du courrier électronique, entre 1987 et 1997 - toute la charge émotive qui pouvait naguère transparaître dans le choix du papier, la couleur de l'encre, la forme de l'écriture, le tremblé d'un mot, les traces de ratures et de larmes éventuelles ${ }^{28}$.

Enfin, le html 1.0, langage originel du Web introduit en 1993, était au départ beaucoup plus préoccupé par l'organisation logique du texte en listes et en hyperliens que par des outils de mise en page. En vertu d'une conception purement abstraite de l'hypertexte, popularisée dans les milieux d'avant-garde dès 1987 avec afternoon de Michael Joyce ${ }^{29}$, le texte devait être dépouillé de toute mise en forme (marges, retraits) et réduit à des segments limités à quelques paragraphes, sans que le lecteur ait même la possibilité d'avoir une vue d'ensemble sur leur agencement ${ }^{30}$. Cela tendait à faire du texte un objet visuellement neutre indexé sur la linéarité de l'oral et réduit à une simple succession de mots. Dès 1994, Roy Harris avait observé lui aussi que la numérisation du texte encourageait une conception extrêmement réductrice de la textualité : «Avec l'ordinateur, on risque de se laisser convaincre que toute l'écriture peut être réduite à un assemblage de points noirs sur un écran $»^{31}$.

Un tel mouvement va nettement à l'encontre d'une évolution millénaire par laquelle le texte est devenu toujours plus tabulaire en intégrant des dispositifs qui permettent au lecteur de contrôler visuel-

\footnotetext{
${ }^{28}$ Voir Melançon B., Sevigne@Internet : remarques sur le courrier électronique et la lettre, Montréal, Fides, 1996. Depuis, les logiciels de courrier se sont dotés de jeux de couleurs et de " papier à lettres ", tandis que les outils de « chat " proposent une riche gamme d'émoticônes pour traduire le ton particulier accompagnant une intervention.

${ }^{29}$ Joyce M., afternoon, a story [cédérom], Cambridge, Eastgate Systems, 1987.

${ }^{30}$ Des tenants de cette conception « intégriste » de l'hypertexte continuent à afficher leur texte au kilomètre et s'étonnent qu'il y ait si peu de « vrais » hypertextes sur le Web : http://www.ucalgary.ca/ dabrent/webliteracies/fewnativ.htm.

${ }^{31}$ Harris R., La sémiologie de l'écriture, Paris, CNRS, 1994, p. 141.
} 
lement la matière à lire, d'en découvrir rapidement les articulations et, grâce aux index et tables des matières, de pouvoir le balayer des yeux à la façon d'un tableau, sans parcours linéaire obligé ${ }^{32}$. Cette tabularité du texte s'est épanouie avec l'apparition des encyclopédies et des ouvrages techniques. Elle trouve son modèle achevé au $\mathrm{XX}^{\mathrm{e}}$ siècle dans les magazines de vulgarisation scientifique, en même temps qu'explosent les recherches sur les divers procédés de traduction visuelle des données. La disposition des informations sous forme de schémas et de graphiques, accompagnés d'illustrations et d'encadrés, encourage une lecture sans ordre imposé, où le lecteur se laisse guider par ses seuls intérêts. C'est aussi au $\mathrm{XX}^{\mathrm{e}}$ siècle qu'on voit se développer le langage des symboles et des logos, dont l'empire est toujours en pleine expansion, et qui couvre un champ toujours plus vaste d'un réel jusqu'ici appréhendé principalement par le langage.

Par rapport à cet idéal de visualisation du texte, l'arrivée massive de l'ordinateur a donc entraîné, dans un premier temps, entre 1982 et 1997, un formidable déficit au plan de la charge émotive et un recul par rapport à la demande du public pour des messages de plus en plus riches au plan visuel. Notre entrée dans la vidéosphère décrite par Régis Debray ${ }^{33}$ était ainsi marquée par de singulières régressions. Il en est résulté dans le public une angoisse diffuse devant la disparition annoncée de l'imprimerie, avec toute la richesse de ses papiers, de ses encres et de ses jeux de caractères : c'est celle-ci que Carson a voulu évoquer dans le titre de son premier ouvrage.

Aujourd'hui, le déficit visuel engendré initialement par l'arrivée de l'ordinateur est en voie de résorption rapide. Le mouvement de fond qui tend à déporter l'activité de lecture vers la visualisation va même en s'accélérant, grâce à la croissance du Web et à la facilité avec laquelle les écrans peuvent accueillir indifféremment texte et images - notamment depuis l'arrivée de logiciels, comme Flash de Macromédia, qui donnent au graphiste une prodigieuse palette de possibilités, comme on peut en voir un exemple dans les poésies

\footnotetext{
${ }^{32}$ Vandendorpe C., Du papyrus à l'hypertexte, Paris, La Découverte, 1999.

${ }^{33}$ Debray R., Vie et mort de l'image, Gallimard, 1992.
} 
graphiques de David Johnston, nouveau calligraphe digital (figure 12).

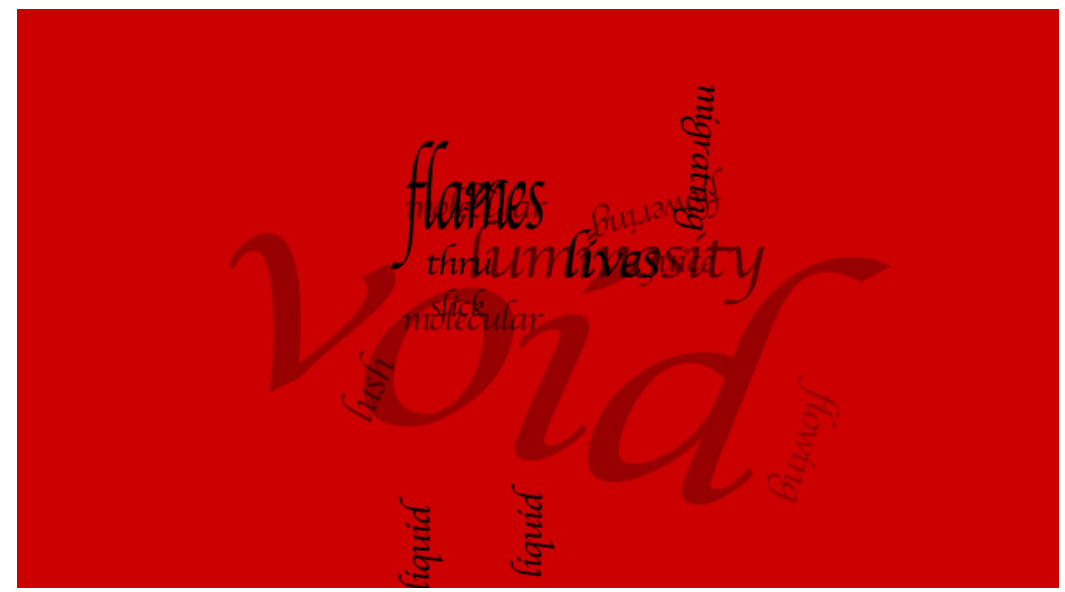

Figure 12. David Johnston. Un instantané tiré de Nomad Lingo.

Décrivant le mouvement général de l'art au $\mathrm{XX}^{\mathrm{e}}$ siècle, Hans Hess note que celui-ci avait consisté pour l'essentiel à donner à l'image une densité conceptuelle en même temps qu'elle s'éloignait toujours plus de la représentation du monde ${ }^{34}$. Il est dès lors paradoxal que le langage écrit, traditionnellement arc-bouté sur la logique et l'abstraction grâce à une typographie transparente, tende maintenant à se rapprocher de l'image. Singulier chassé-croisé de nos outils de formalisation mentale sous la pression des tendances opposées de la culture savante et de la culture populaire.

Mallarmé avait décroché le langage du référent et rompu l'alliance millénaire entre les mots et les choses ${ }^{35}$. Le mouvement qui anime aujourd'hui les écoles de graphisme, particulièrement en Angleterre et aux Etats-Unis, tend à décrocher la typographie du langage et à la rapprocher du monde des images afin de la rendre apte à communiquer la richesse et la chaleur des émotions. Mais alors que les ima-

\footnotetext{
${ }^{34}$ Hess H., How pictures mean, New York, Pantheon Books, 1974.

${ }^{35}$ Steiner G., Réelles présences, Paris, Gallimard, 1989, p. 123.
} 
ges présentent une fluidité de sens qui décourage l'interprétation, le texte transformé en image peut toujours être récupéré en significations grâce à sa nature verbale et à la surdétermination de ses composantes typographiques. Le texte se trouve ainsi au croisement de deux mondes, jouissant à la fois de la séduction visuelle de l'image et de la promesse d'un sens à déchiffrer. Il est comme un spectacle à lire, une suite d'hiéroglyphes qui contiendraient en elles-mêmes les clés de leur déchiffrement - pour peu que le lecteur accepte d'examiner sous l'angle du lisible ce qui apparaît d'abord comme pure visibilité. 